\title{
Generalized Measures in Gauge Theory
}

\author{
John C. Baez \\ Department of Mathematics \\ University of California \\ Riverside CA 92521
}

October 30, 1993

\begin{abstract}
Let $P \rightarrow M$ be a principal $G$-bundle. We construct well-defined substitutes for "Lebesgue measure" on the space $\mathcal{A}$ of connections on $P$ and for "Haar measure" on the group $\mathcal{G}$ of gauge transformations. More precisely, we define algebras of "cylinder functions" on the spaces $\mathcal{A}, \mathcal{G}$, and $\mathcal{A} / \mathcal{G}$, and define generalized measures on these spaces as continuous linear functionals on the corresponding algebras. Borrowing some ideas from lattice gauge theory, we characterize generalized measures on $\mathcal{A}, \mathcal{G}$, and $\mathcal{A} / \mathcal{G}$ in terms of graphs embedded in $M$. We use this characterization to construct generalized measures on $\mathcal{A}$ and $\mathcal{G}$, respectively. The "uniform" generalized measure on $\mathcal{A}$ is invariant under the group of automorphisms of $P$. It projects down to the generalized measure on $\mathcal{A} / \mathcal{G}$ considered by Ashtekar and Lewandowski in the case $G=S U(n)$. The "generalized Haar measure" on $\mathcal{G}$ is right- and left-invariant as well as $\operatorname{Aut}(P)$-invariant. We show that averaging any generalized measure on $\mathcal{A}$ against generalized Haar measure gives a $\mathcal{G}$-invariant generalized measure on $\mathcal{A}$.
\end{abstract}

\section{Introduction}

The space $\mathcal{A}$ of connections on a principal bundle is an infinite-dimensional affine space, and the while the notion of the "Lebesgue measure" $\mathcal{D} A$ on $\mathcal{A}$ has been very fruitful, it is mathematically ill-defined. Some of the infinities in quantum field theory calculations can be avoided by projecting down $\mathcal{D} A$ to the space $\mathcal{A} / \mathcal{G}$ of connections modulo gauge transformations, but certainly not all. While the theory of cylinder measures on infinite-dimensional vector spaces [6] provides a rigorous framework for interpreting the Gaussian "measures" appearing in the physics of the free boson field [5], it is usually quite difficult to apply this theory to the study of gauge fields, except in the case of 2d Yang-Mills theory. It is thus desirable to generalize the concept of measure in a manner more suited to the needs of gauge theory.

Recently Ashtekar and Isham [1] have proposed an approach based on the idea of a Wilson loop, that is, the trace of the holonomy of a connection around a loop in the base manifold $M$. Wilson loops are very natural observables in gauge theory, and in the "loop representation" of gauge theories they play a primary role [7]. In the case 
of the group $G=S U(n)$, Ashtekar and Lewandowski [2] used this approach to define and construct a very natural "generalized measure" $\mu_{\mathrm{AL}}$ on $\mathcal{A} / \mathcal{G}$, which is invariant under diffeomorphisms of $M$. The author [3] extended this approach to construct a rich variety of diffeomorphism-invariant generalized measures on $\mathcal{A} / \mathcal{G}$ when $G$ is compact. These generalized measures give rise to invariants of multiloops (collections of loops) in the base manifold, and their classification involves a combination of singularity theory and knot theory.

In this paper we show that one can define generalized measures on $\mathcal{A}$. All of these project down to generalized measures on $\mathcal{A} / \mathcal{G}$, but even when one is interested in gauge-invariant quantities, it is sometimes easier to work "upstairs" on $\mathcal{A}$. In particular, when $G$ is compact, there is a "uniform" generalized measure $\mu_{u}$ on $\mathcal{A}$ that projects down to $\mu_{A L}$ under the map $\mathcal{A} \rightarrow \mathcal{A} / \mathcal{G}$. This generalized measure $\mu_{u}$ is in some respects a rigorous substitute for the ill-defined "Lebesgue measure" on $\mathcal{A}$, but it is actually built using Haar measure on $G$. We also define generalized measures on $\mathcal{G}$, and when $G$ is compact we construct a natural example $\mu_{\mathrm{H}}$ that is a rigorous substitute for Haar measure on $\mathcal{G}$. As an application of this "generalized Haar measure" we show that any generalized measure on $\mathcal{A}$ can be averaged against $\mu_{\mathrm{H}}$ to give a $\mathcal{G}$-invariant generalized measure on $\mathcal{A}$.

We emphasize that these constructions are no panacea; in particular, they are unlikely to be of much use in 4-dimensional Yang-Mills theory, where one expects that only "smeared" Wilson loops will serve as physical observables [4]. With some modification, these constructions might allow the construction of the Chern-Simons path integral as a generalized measure, as discussed in [3]. They may also be suited for rigorous work on the loop representation of quantum gravity [7, 8].

\section{Generalized Measures}

Let $M$ be a manifold, possibly with boundary, let $G$ be a Lie group, and let $\pi: P \rightarrow M$ be a principal $G$-bundle. Let $\mathcal{A}$ be the space of connections on $P$ and let $\mathcal{G}$ be the group of gauge transformations. Let $\operatorname{Diff}(M)$ denote the group of diffeomorphisms of $M$ restricting to diffeomorphisms of $\partial M$. Everything in this section applies equally to the following three cases:

1. The $C^{\infty}$ case: $M$ and $P$ are smooth, $\pi$ is smooth, and $\mathcal{A}, \mathcal{G}$, and $\operatorname{Diff}(M)$ consist of smooth connections, gauge transformations, and diffeomorphisms, respectively.

2. The $C^{\omega}$ case: $M$ and $P$ are real-analytic, $\pi$ is real-analytic, and $\mathcal{A}, \mathcal{G}$, and $\operatorname{Diff}(M)$ consist of real-analytic connections, gauge transformations, and diffeomorphisms, respectively.

3. The hybrid case: $M$ is real-analytic and $P$ is smooth, $\pi$ is smooth, $\mathcal{A}$ and $\mathcal{G}$ consist of smooth connections and gauge transformations, respectively, and 
$\operatorname{Diff}(M)$ consists of real-analytic diffeomorphisms.

We will define sub- $\mathrm{C}^{*}$-algebras of the bounded continuous complex functions on $\mathcal{A}, \mathcal{G}$, and $\mathcal{A} / \mathcal{G}$ (with their $C^{\infty}$ topologies). By a "generalized measure" on one of these spaces we will mean simply a continuous linear functional on the corresponding $\mathrm{C}^{*}$-algebra. Every finite regular Borel measure on one of these spaces defines such a generalized measure, but the most interesting generalized measures are not of this form. It is easiest to construct generalized measures in case 3 above, so in the next section we will restrict attention to that case, even though case 1 is in some ways the most natural.

Given a path $\gamma:[0,1] \rightarrow M$, let $\mathcal{A}_{\gamma}$ be the space of all maps from the fiber $P_{\gamma(0)}$ to the fiber $P_{\gamma(1)}$ that can be obtained as holonomies along $\gamma$ of some connection $A \in \mathcal{A}$. Note that there is a natural map

$$
\begin{aligned}
p_{\gamma}: \mathcal{A} & \rightarrow \mathcal{A}_{\gamma} \\
A & \mapsto A_{\gamma}
\end{aligned}
$$

assigning to each connection $A \in \mathcal{A}$ its holonomy $A_{\gamma}$ along $\gamma$. One should think of $p_{\gamma}: A \mapsto A_{\gamma}$ as picking out a small piece of information about the connection $A$; one can reconstruct $A$ from all the pieces $\left\{A_{\gamma}\right\}$. Fixing trivializations of $P$ at the endpoints $\gamma(0)$ and $\gamma(1), \mathcal{A}_{\gamma}$ can be identified with an open and closed subspace of the group $G$. We give $\mathcal{A}_{\gamma}$ the subspace topology (which is independent of the choice of trivialization). This makes the map $p_{\gamma}$ continuous.

We say $f$ is a cylinder function on $\mathcal{A}$ if it is of the form

$$
f(A)=F\left(A_{\gamma_{1}}, \ldots, A_{\gamma_{n}}\right),
$$

where $\left\{\gamma_{i}\right\}$ is a finite set of paths in $M$ (required to be real-analytic in the $C^{\omega}$ and hybrid cases) and

$$
F: \prod_{i} \mathcal{A}_{\gamma_{i}} \rightarrow \mathbf{C}
$$

is a bounded continuous function. Let $\operatorname{Fun}_{0}(\mathcal{A})$ denote the space of cylinder functions on $\mathcal{A}$, which is a $*$-subalgebra of the bounded continuous functions on $\mathcal{A}$. The completion of $\operatorname{Fun}_{0}(\mathcal{A})$ with respect to the sup norm, which we denote by $\operatorname{Fun}(\mathcal{A})$, is thus a $\mathrm{C}^{*}$-subalgebra of the bounded continuous functions on $\mathcal{A}$.

Note that $\mathcal{G}$ acts as $*$-automorphisms of $\operatorname{Fun}_{0}(\mathcal{A})$ by

$$
g f(A)=f\left(g^{-1} A\right) .
$$

The $\mathcal{G}$-invariant functions in $\operatorname{Fun}_{0}(\mathcal{A})$ may be regarded as functions on $\mathcal{A} / \mathcal{G}$, and we denote the algebra of all such functions as $\operatorname{Fun}_{0}(\mathcal{A} / \mathcal{G})$. We call these cylinder functions on $\mathcal{A} / \mathcal{G}$. We denote the completion of $\operatorname{Fun}_{0}(\mathcal{A} / \mathcal{G})$ with respect to the sup norm by $\operatorname{Fun}(\mathcal{A} / \mathcal{G})$. This may be regarded either as a $\mathrm{C}^{*}$-subalgebra of the bounded continuous 
functions on $\mathcal{A} / \mathcal{G}$ (with its quotient topology), or of the bounded continuous $\mathcal{G}$ invariant functions on $\mathcal{A}$. It can be seen that as special cases of this algebra one obtains the "holonomy $\mathrm{C}^{*}$-algebra" defined in the smooth case by Ashtekar and Isham [1] for $G=S U(2)$ and the "analytic holonomy $\mathrm{C}^{*}$-algebra" defined in the hybrid case by Ashtekar and Lewandowski [2] for $G=S U(n)$.

We may also define cylinder functions on the group $\mathcal{G}$ of gauge transformations. Given a point $x \in M$, let $\mathcal{G}_{x}$ be the fiber at $x$ of the bundle $P \times_{\text {Ad }} G$, with its subspace topology. An element $g \in \mathcal{G}$ is a section of $P \times_{\mathrm{Ad}} G$, so there is a natural map

$$
\begin{aligned}
p_{x}: \mathcal{G} & \rightarrow \mathcal{G}_{x} \\
g & \mapsto g_{x}
\end{aligned}
$$

One should think of $p_{x}: g \mapsto g_{x}$ as picking out a small piece of information about the gauge transformation $g$; one can reconstruct $g$ from the pieces $\left\{g_{x}\right\}$. Note that $\mathcal{G}_{x}$ is naturally a group, and that $p_{x}$ is a homomorphism.

We say $f$ is a cylinder function on $\mathcal{G}$ if it is of the form

$$
f(g)=F\left(g_{x_{1}}, \ldots, g_{x_{n}}\right),
$$

where $\left\{x_{i}\right\}$ is a finite set of points in $M$ and

$$
F: \prod_{i} \mathcal{G}_{\gamma_{i}} \rightarrow \mathbf{C}
$$

is bounded and continuous. The completion of the algebra $\operatorname{Fun}_{0}(\mathcal{G})$ of cylinder functions on $\mathcal{G}$ is a $\mathrm{C}^{*}$-subalgebra of the bounded continuous functions on $\mathcal{G}$, which we denote by $\operatorname{Fun}(\mathcal{G})$.

By a generalized measure on $\mathcal{A}, \mathcal{G}$, or $\mathcal{A} / \mathcal{G}$ we mean a continuous linear functional on $\operatorname{Fun}(\mathcal{A}), \operatorname{Fun}(\mathcal{G})$, or $\operatorname{Fun}(\mathcal{A} / \mathcal{G})$, respectively. Note that every generalized measure on $\mathcal{A}$ "projects down" to a generalized measure on $\mathcal{A} / \mathcal{G}$; this operation of "projecting down" is really just restriction of a continuous linear functional on $\operatorname{Fun}(\mathcal{A})$ to the subalgebra $\operatorname{Fun}(\mathcal{A} / \mathcal{G})$.

Let $\operatorname{Aut}(P)$ denote the group of bundle automorphisms $g$ such that for some $h \in \operatorname{Diff}(M), \pi(g(p))=h(\pi(p))$ for all $p \in P$. (In cases 2 and 3, recall that $h$ must be real-analytic.) Then we have an exact sequence

$$
1 \rightarrow \mathcal{G} \rightarrow \operatorname{Aut}(P) \rightarrow \operatorname{Diff}(M) \rightarrow 1 .
$$

The group $\operatorname{Aut}(P)$ acts as $*$-automorphisms of $\operatorname{Fun}(\mathcal{A})$ by

$$
g f(A)=f\left(g^{-1} A\right) .
$$

so we obtain an action of $\operatorname{Diff}(M)$ as $*$-automorphisms of $\operatorname{Fun}(\mathcal{A} / \mathcal{G})$. By duality, $\operatorname{Aut}(P)$ acts on the generalized measures on $\mathcal{A}$, and $\operatorname{Diff}(M)$ acts on the generalized measures on $\mathcal{A} / \mathcal{G}$. Any $\operatorname{Aut}(P)$-invariant generalized measure on $\mathcal{A}$ projects down to a $\operatorname{Diff}(M)$-invariant generalized measure on $\mathcal{A} / \mathcal{G}$. Note also that $\operatorname{Aut}(P)$ acts on the generalized measures on $\mathcal{G}$, as do left and right translation. 


\section{Characterizing Generalized Measures}

In this section and the next we restrict our attention to the "hybrid case," case 2 of the previous section. Ashtekar and Lewandowski recognized the importance of this case when they used it to construct a very natural sort of Diff( $M)$-invariant generalized measure on $\mathcal{A} / \mathcal{G}$ for $G=S U(2)$. Subsequently they, and independently the author, were able to generalize this construction to more general compact Lie groups, and also to give a rather concrete characterization of all generalized measures on $\mathcal{A} / \mathcal{G}$. In addition, the author has given a recipe for constructing many $\operatorname{Diff}(M)$-invariant examples of such generalized measures.

Here we give concrete characterizations of generalized measures on $\mathcal{A}, \mathcal{G}$, and $\mathcal{A} / \mathcal{G}$ when $G$ is any Lie group. First, we need a notion of an embedded graph in $M$, a slight variant of that in [3]. We define an embedded graph $\phi$ in $M$ to be a finite collection of real-analytic paths $\phi_{j}:[0,1] \rightarrow M$ such that:

1. for all $j, \phi_{j}$ is one-to-one,

2. for all $j,\left.\phi_{j}\right|_{(0,1)}$ is an embedding,

3. for all $j$ and $k, \phi_{j}[0,1] \cap \phi_{k}[0,1] \subseteq\left\{\phi_{j}(0), \phi_{j}(1)\right\}$.

The paths $\phi_{j}$ are called the edges of $\phi$, and the points $\phi_{j}(0), \phi_{j}(1)$ are called the vertices of $\phi$. Somewhat redundantly, we write $E(\phi)$ for the set of edges of $\phi$ and $V(\phi)$ for the set of (distinct) vertices. Note that the set

$$
|\phi|=\bigcup_{j} \phi_{j}[0,1] \subseteq M
$$

equipped with the subspace topology indeed has the topology of a finite graph.

The following lemma proved by Ashtekar and Lewandowsi plays a key technical role.

Lemma 1. 2] Let $\left\{\gamma_{i}\right\}$ be a finite collection of real-analytic paths in $M$. Then there exists an embedded graph $\phi$ such that for each $\gamma_{i}$ there exist paths in $\phi$ such that $\gamma_{i}$ is equivalent to a product of these paths and their inverses, up to a continuous orientation-preserving reparametrization.

Given an analytic graph $\phi$ in $M$, let

$$
\mathcal{A}_{\phi}=\prod_{\gamma \in E(\phi)} \mathcal{A}_{\gamma}
$$

the Cartesian product over all edges $\gamma$ of $\phi$ of the spaces $\mathcal{A}_{\gamma}$, equipped with the product topology. Similarly, let

$$
\mathcal{G}_{\phi}=\prod_{x \in V(\phi)} \mathcal{G}_{x}
$$


equipped with the product topology. We may write any element of $\mathcal{G}_{\phi}$ as a tuple $\left(g_{x}\right)_{x \in V(\phi)}$ where $g_{x} \in \mathcal{G}_{x}$. Similarly, we may write any element of $\mathcal{A}_{\phi}$ as a tuple $\left(A_{\gamma}\right)_{\gamma \in E(\phi)}$, where $A_{\gamma}: P_{\gamma(0)} \rightarrow P_{\gamma(1)}$. There are natural maps $p_{\phi}: \mathcal{A} \rightarrow \mathcal{A}_{\phi}$ and $p_{\phi}: \mathcal{G} \rightarrow$ $\mathcal{G}_{\phi}$, given by

$$
\begin{aligned}
p_{\phi}(A) & =\left(A_{\gamma}\right)_{\gamma \in E(\phi)}, \\
p_{\phi}(g) & =\left(g_{x}\right)_{x \in V(\phi)} .
\end{aligned}
$$

Though we denote both of these maps by $p_{\phi}$, the meaning should be clear from context. Both these maps are onto, since we can always find a connection having any specified holonomies in the sets $\mathcal{A}_{\phi}$, and we can always find a gauge transformation having any specified values at the vertices of $\phi$. Given $A \in \mathcal{A}$ and $g \in \mathcal{G}$, we will sometimes write $A_{\phi}$ for $p_{\phi}(A)$ and $g_{\phi}$ for $p_{\phi}(g)$.

In the above we are borrowing an idea from lattice gauge theory, in which "connections" assign group elements to the edges of a lattice, while "gauge transformations" assign group elements to vertices. We can make this analogy very precise if the group $G$ is connected. In this cases, we can trivialize $P$ over $|\phi|$ for any embedded graph $\phi$. Fixing a trivialization gives an identification of $\mathcal{A}_{\gamma}$, for any edge $\gamma$ of $\phi$, with the group $G$, hence

$$
\mathcal{A}_{\phi} \cong G^{E(\phi)} .
$$

Similarly, fixing a trivialization gives an identification of $\mathcal{G}_{x}$, for any vertex $x$ of $\phi$, with $G$, so

$$
\mathcal{G}_{\phi} \cong G^{V(\phi)} .
$$

The group $\mathcal{G}_{\phi}$ acts on the space $\mathcal{A}_{\phi}$ as follows:

$$
\left(g_{x}\right)_{x \in V(\phi)}\left(A_{\gamma}\right)_{\gamma \in E(\phi)}=\left(g_{\gamma(1)} A_{\gamma} g_{\gamma(0)}^{-1}\right)_{\gamma \in E(\phi)} .
$$

This action is compatible with the action of $\mathcal{G}$ on $\mathcal{A}$, as follows:

$$
p_{\phi}(g) p_{\phi}(A)=p_{\phi}(g A)
$$

for any $g \in \mathcal{G}, A \in \mathcal{A}$.

Let $\operatorname{Fun}\left(\mathcal{A}_{\phi}\right)$ denote the algebra of bounded continuous functions on $\mathcal{A}_{\phi}$. We will identify $F \in \operatorname{Fun}\left(\mathcal{A}_{\phi}\right)$ with the function $f$ on $\mathcal{A}$ given by

$$
f(A)=F\left(A_{\phi}\right),
$$

allowing us to write

$$
\operatorname{Fun}\left(\mathcal{A}_{\phi}\right) \subseteq \operatorname{Fun}_{0}(\mathcal{A}) .
$$

Since a generalized measure $\mu$ on $\mathcal{A}$ is just a continuous linear functional on $\operatorname{Fun}(\mathcal{A})$, we can restrict $\mu$ to a continuous linear functional $\mu_{\phi}$ on $\operatorname{Fun}\left(\mathcal{A}_{\phi}\right)$. (When $G$ is compact, $\mathcal{A}_{\phi}$ is compact, so the Riesz-Markov theorem allows us to identify continuous linear functionals on $\operatorname{Fun}\left(\mathcal{A}_{\phi}\right)$ with finite regular Borel measures on $\mathcal{A}_{\phi}$.) A set of 
continuous linear functionals $\mu_{\phi} \in \operatorname{Fun}\left(\mathcal{A}_{\phi}\right)^{*}$, one for each embedded graph $\phi$, will be called a family. The following theorem gives necessary and sufficient conditions for a family $\left\{\mu_{\phi}\right\}$ to come from a generalized measure on $\mathcal{A}$. As in [3], these conditions can be used to construct concrete examples of generalized measures.

Given embedded graphs $\phi, \psi$, we say that $\phi$ is included in $\psi$, which we write as $\phi \hookrightarrow \psi$, if every edge of $\phi$ is, up to orientation-preserving reparametrization, a product of edges of $\psi$ and their inverses. Note that $\phi \hookrightarrow \psi$ implies that every vertex of $\phi$ is a vertex of $\psi$, and that $|\phi| \subseteq|\psi|$. It also implies that $\operatorname{Fun}\left(\mathcal{A}_{\phi}\right) \subseteq \operatorname{Fun}\left(\mathcal{A}_{\psi}\right)$. We say that the family $\left\{\mu_{\phi}\right\}$ is consistent if $\phi \hookrightarrow \psi$ implies that the restriction of $\mu_{\psi}$ to $\operatorname{Fun}\left(\mathcal{A}_{\phi}\right)$ is $\mu_{\phi}$. We say that the family $\left\{\mu_{\phi}\right\}$ is uniformly bounded if there is a constant $C>0$ such that $\left\|\mu_{\phi}\right\|<C$ for all $\phi$.

Theorem 1. Suppose $\mu$ is a generalized measure on $\mathcal{A}$, that is, a continuous linear functional on $\operatorname{Fun}(\mathcal{A})$. For any embedded graph $\phi$ in $M$, let $\mu_{\phi}$ denote the restriction of $\mu$ to $\operatorname{Fun}\left(\mathcal{A}_{\phi}\right)$. Then $\left\{\mu_{\phi}\right\}$ is a consistent and uniformly bounded family. Conversely, if $\left\{\mu_{\phi}\right\}$ is a consistent and uniformly bounded family, there is a unique generalized measure $\mu$ on $\mathcal{A}$ whose restriction to $\operatorname{Fun}\left(\mathcal{A}_{\phi}\right)$ is $\mu_{\phi}$.

Proof - If $\mu$ is a generalized measure on $\mathcal{A}$ the family $\left\{\mu_{\phi}\right\}$ obtained by restriction is consistent, and $\left\|\mu_{\phi}\right\| \leq\|\mu\|$, so it is uniformly bounded. Conversely, suppose we are given a consistent and uniformly bounded family $\left\{\mu_{\phi}\right\}$. We first define a linear functional $\mu$ on $\operatorname{Fun}_{0}(\mathcal{A})$ as follows. Any element $f \in \operatorname{Fun}_{0}(\mathcal{A})$ is of the form

$$
f(A)=F\left(A_{\gamma_{1}}, \ldots, A_{\gamma_{n}}\right),
$$

where $\left\{\gamma_{i}\right\}$ are paths in $M$. In this situation we say that $f$ can be expressed in terms of the paths $\left\{\gamma_{i}\right\}$. Construct an embedded graph $\phi$ from the paths $\gamma_{i}$ as in Lemma 1. Then $f \in \operatorname{Fun}\left(\mathcal{A}_{\phi}\right)$. Define

$$
\mu(f)=\mu_{\phi}(f) .
$$

We need to check that $\mu$ is well-defined, linear, and extends to a continuous linear functional on $\operatorname{Fun}(\mathcal{A})$. If the extension exists, it is unique, since $\operatorname{Fun}_{0}(A)$ is dense in $\operatorname{Fun}(\mathcal{A})$.

For well-definedness, suppose that $f$ can be expressed in two ways, in terms of paths $\left\{\gamma_{i}\right\}$ or in terms of paths $\left\{\gamma_{j}^{\prime}\right\}$. Using Lemma 1, construct embedded graphs $\phi$ from the paths $\left\{\gamma_{i}\right\}, \phi^{\prime}$ from the paths $\left\{\gamma_{j}^{\prime}\right\}$, and $\psi$ from the paths $\left\{\gamma_{i}, \gamma_{j}^{\prime}\right\}$. Note that $\phi \hookrightarrow \psi$ and $\phi^{\prime} \hookrightarrow \psi$. Thus

$$
\mu_{\phi}(f)=\mu_{\psi}(f)=\mu_{\phi^{\prime}}(f) .
$$

For linearity, suppose $f, g \in \operatorname{Fun}_{0}(\mathcal{A})$. Then $f+g \in \operatorname{Fun}_{0}(\mathcal{A})$ and there exist paths $\left\{\gamma_{i}\right\}$ in terms of which $f, g$, and $f+g$ can all be expressed. Using Lemma 1 , construct an embedded graph $\phi$ from the paths $\left\{\gamma_{i}\right\}$. Then

$$
\mu(f+g)=\mu_{\phi}(f+g)=\mu_{\phi}(f)+\mu_{\phi}(g)=\mu(f)+\mu(g) .
$$


Clearly $\mu(\lambda f)=\lambda \mu(f)$ for all $\lambda \in \mathbf{C}$.

Finally, to show that $\mu$ extends to a continuous linear functional on $\operatorname{Fun}(\mathcal{A})$ it suffices to note that there exists $C>0$ with $\|\mu(f)\| \leq C\|f\|$ for any $f \in \operatorname{Fun}_{0}(\mathcal{A})$, by the uniform boundedness of the family $\left\{\mu_{\phi}\right\}$.

Completely analogous results holds for generalized measures on $\mathcal{G}$ and $\mathcal{A} / \mathcal{G}$. Let $\operatorname{Fun}\left(\mathcal{A}_{\phi} / G_{\phi}\right)$ denote the subalgebra of $\operatorname{Fun}\left(\mathcal{A}_{\phi}\right)$ consisting of functions invariant under the action of $\mathcal{G}_{\phi}$. Alternatively, $\operatorname{Fun}\left(\mathcal{A}_{\phi} / \mathcal{G}_{\phi}\right)$ may be regarded as the algebra of bounded continuous functions on $\mathcal{A}_{\phi} / \mathcal{G}_{\phi}$, equipped with its quotient topology. A generalized measure $\mu$ on $\mathcal{A} / \mathcal{G}$ restricts to a family of elements $\mu_{\phi} \in \operatorname{Fun}\left(\mathcal{A}_{\phi} / \mathcal{G}_{\phi}\right)^{*}$, one for each embedded graph $\phi$. (When $G$ is compact these are the same as finite regular Borel measures on $\mathcal{A}_{\phi} / \mathcal{G}_{\phi}$.) We define consistency and uniform boundedness of such families as before, and obtain:

Theorem 2. Suppose $\mu$ is a generalized measure on $\mathcal{A} / \mathcal{G}$. For any embedded graph $\phi$ in $M$, let $\mu_{\phi}$ denote the restriction of $\mu$ to $\operatorname{Fun}\left(\mathcal{A}_{\phi} / \mathcal{G}_{\phi}\right)$. Then $\left\{\mu_{\phi}\right\}$ is a consistent and uniformly bounded family. Conversely, if $\left\{\mu_{\phi}\right\}$ is a consistent and uniformly bounded family, there is a unique generalized measure $\mu$ on $\mathcal{A} / \mathcal{G}$ whose restriction to $\operatorname{Fun}\left(\mathcal{A}_{\phi} / \mathcal{G}_{\phi}\right)$ is $\mu_{\phi}$.

Proof - The proof follows that of Theorem [1.

Let $\operatorname{Fun}\left(\mathcal{G}_{\phi}\right)$ denote the algebra of bounded continuous functions on $\mathcal{G}_{\phi}$. We will identify $F \in \operatorname{Fun}\left(\mathcal{G}_{\phi}\right)$ with the function $f$ on $\mathcal{G}$ given by $f(g)=F\left(g_{\phi}\right)$, allowing us to write

$$
\operatorname{Fun}\left(\mathcal{G}_{\phi}\right) \subseteq \operatorname{Fun}_{0}(\mathcal{G})
$$

Thus a generalized measure $\mu$ on $\mathcal{G}$ restricts to a family $\left\{\mu_{\phi}\right\}$ of elements of $\operatorname{Fun}\left(\mathcal{G}_{\phi}\right)^{*}$. (When $G$ is compact, elements of $\operatorname{Fun}\left(\mathcal{G}_{\phi}\right)^{*}$ are the same as finite regular Borel measures on $\mathcal{G}_{\phi}$.) We define consistency and uniform boundedness of such families as in the case of $\mathcal{A}$.

Theorem 3. Suppose $\mu$ is a generalized measure on $\mathcal{G}$. For any embedded graph $\phi$ in $M$, let $\mu_{\phi}$ denote the restriction of $\mu$ to $\operatorname{Fun}\left(\mathcal{G}_{\phi}\right)$. Then $\left\{\mu_{\phi}\right\}$ is a consistent and uniformly bounded family. Conversely, if $\left\{\mu_{\phi}\right\}$ is a consistent and uniformly bounded family, there is a unique generalized measure $\mu$ on $\mathcal{G}$ whose restriction to $\operatorname{Fun}\left(\mathcal{G}_{\phi}\right)$ is $\mu_{\phi}$.

Proof - The proof follows that of Theorem 1.

We conclude with an alternate description of $\operatorname{Fun}(\mathcal{A} / \mathcal{G})$. Recall that functions in $\operatorname{Fun}(\mathcal{A} / \mathcal{G})$ may be regarded as limits of $\mathcal{G}$-invariant cylinder functions on $\mathcal{A}$. At least when $G$ is amenable (for example, compact, abelian, or an extension of an amenable group by an amenable group), these are precisely the same as $\mathcal{G}$-invariant functions on $\mathcal{A}$ that are limits of cylinder functions: 
Theorem 4. Suppose that $G$ is amenable. Then $\operatorname{Fun}(\mathcal{A} / \mathcal{G})$ is equal to the subalgebra of $\mathcal{G}$-invariant functions in $\operatorname{Fun}(\mathcal{A})$.

Proof - It is immediate that elements of $\operatorname{Fun}(\mathcal{A} / \mathcal{G})$ are $\mathcal{G}$-invariant and lie in $\operatorname{Fun}(\mathcal{A})$. To prove the opposite inclusion, suppose $f \in \operatorname{Fun}(\mathcal{A})$ is $\mathcal{G}$-invariant. Then there exists a sequence $f_{i} \in \operatorname{Fun}_{0}(\mathcal{A})$ with $f_{i} \rightarrow f$. To show $f \in \operatorname{Fun}(\mathcal{A} / \mathcal{G})$ it suffices to show the existence of a sequence of $\mathcal{G}$-invariant elements of $\operatorname{Fun}_{0}(\mathcal{A})$ converging to $f$. We may suppose $f_{i} \in \operatorname{Fun}\left(\mathcal{A}_{\phi_{i}}\right)$. The group $\mathcal{G}_{\phi_{i}}$, being isomorphic to a product of copies of $\mathcal{G}$, is amenable. Let $M_{i}: \operatorname{Fun}(\mathcal{A}) \rightarrow \operatorname{Fun}(\mathcal{A})$ denote the result of averaging over the action of $\mathcal{G}_{\phi_{i}}$ with respect to an invariant mean. Noting that $M_{i}: \operatorname{Fun}\left(\mathcal{A}_{\phi_{i}}\right) \rightarrow$ $\operatorname{Fun}\left(\mathcal{A}_{\phi_{i}}\right)$, that $M_{i}$ is a contraction, and that $M_{i} f=f$, we conclude that $M_{i} f_{i}$ is a sequence of $\mathcal{G}$-invariant elements of $\operatorname{Fun}_{0}(\mathcal{A})$ converging to $f$.

It is not clear whether the hypothesis of amenability is necessary.

\section{Examples}

Now we consider the case where $G$ is compact. In this case we construct a generalized measure on $\mathcal{A}$ that we call the uniform generalized measure. This generalized measure is invariant under all of $\operatorname{Aut}(P)$. It thus projects down to a generalized measure on $\mathcal{A} / \mathcal{G}$ that is $\operatorname{Diff}(M)$-invariant, as described at the end of Section 2. The result is the generalized measure on $\mathcal{A} / \mathcal{G}$ constructed for $G=S U(n)$ by Ashtekar and Lewandowski [2]. We also construct a generalized measure on $\mathcal{G}$ called generalized Haar measure, which is both left- and right-invariant as well as $\operatorname{Aut}(P)$-invariant. We also show how to average any generalized measure on $\mathcal{A}$ against generalized Haar measure on $\mathcal{G}$ to obtain a $\mathcal{G}$-invariant generalized measure on $\mathcal{A}$.

As in the previous section, we assume $P \mapsto M$ is a $G$-principal bundle and work in the "hybrid case," case 2 of Section 2. Let $m$ denote normalized Haar measure on $G$, which is assumed compact. Suppose $\gamma$ is any path in $M$. Fixing a trivialization of $P$ at $\gamma(0)$ and $\gamma(1)$ we obtain an identification of $\mathcal{A}_{\gamma}$ with a closed and open subset $X \subseteq G$. Define the measure $\mu_{\gamma}$ on $\mathcal{A}_{\gamma}$ to be the restriction of $m$ to $\mathcal{A}_{\gamma}$. A change of trivialization of $P$ at $\gamma(0)$ changes $X \subseteq G$ by a left translation, while a change of trivialization at $\gamma(1)$ changes $X$ by a right translation. Since $m$ is left- and rightinvariant, it follows that $\mu_{\gamma}$ is independent of the choice of trivializations at $\gamma(0)$ and $\gamma(1)$. Recall that for any embedded graph $\phi$

$$
\mathcal{A}_{\phi}=\prod_{\gamma \in E(\phi)} \mathcal{A}_{\gamma} .
$$

Define $\mu_{\phi}$ to be the finite regular Borel measure on $\mathcal{A}_{\phi}$ given by the product of the measures $\mu_{\gamma}$. Note that

$$
\left\|\mu_{\phi}\right\| \leq 1
$$


for all $\phi$. One can show that $\left\{\mu_{\phi}\right\}$ is a consistent family in the sense of Theorem 1] (for details, see [2, 3]). Theorem 1 thus implies the existence of a unique generalized measure $\mu$ on $\mathcal{A}$ such that for all $\phi, \mu_{\phi}$ is the restriction of $\mu$ to $\operatorname{Fun}\left(\mathcal{A}_{\phi}\right)$. We call this generalized measure $\mu_{u}$ the uniform generalized measure on $\mathcal{A}$. It is easily seen from the natural way in which it was constructed that $\mu_{u}$ is $\operatorname{Aut}(P)$-invariant, and thus projects down to a $\operatorname{Diff}(M)$-invariant generalized measure $\mu_{\mathrm{AL}}$ on $\mathcal{A} / \mathcal{G}$. We call $\mu_{\mathrm{AL}}$ the Ashtekar-Lewandowski generalized measure on $\mathcal{A} / \mathcal{G}$.

Similarly, given any point $x \in M$, a trivialization of $P$ at $x$ gives an identification of $\mathcal{G}_{x}$ with $G$. Using this identification the Haar measure $m$ on $G$ gives rise to a measure $\mu_{x}$ on $\mathcal{G}_{x}$. Since $m$ is left- and right-invariant, $\mu_{x}$ independent of the choice of trivialization of $P$ at $x$. Define the finite regular Borel measure $\mu_{\phi}$ on

$$
\mathcal{G}_{\phi}=\prod_{x \in V(\phi)} \mathcal{G}_{x}
$$

to be the product of the measures $\mu_{x}$. The family $\left\{\mu_{\phi}\right\}$ is consistent and uniformly bounded, so by Theorem 3 there is a unique generalized measure $\mu_{\mathrm{H}}$ on $\mathcal{G}$ such that for all $\phi, \mu_{\phi}$ is the restriction of $\mu_{\mathrm{H}}$ to $\operatorname{Fun}\left(\mathcal{G}_{\phi}\right)$. We call this generalized measure generalized Haar measure on $\mathcal{G}$. By naturality, $\mu_{\mathrm{H}}$ is invariant under the action of $\operatorname{Aut}(P)$ on $\mathcal{G}$. By the invariance properties of Haar measure, $\mu_{\mathrm{H}}$ is also left- and right-invariant. That is, the left and right actions of $\mathcal{G}$ on itself give rise to actions of $\mathcal{G}$ on $\operatorname{Fun}(\mathcal{G})$, hence dually on $\operatorname{Fun}(\mathcal{G})^{*}$, and $\mu_{\mathrm{H}}$ is preserved by these actions.

We can convolve generalized measures on $\mathcal{G}$ as follows. For each embedded graph $\phi$ one can convolve finite regular Borel measures on the compact Lie group $\mathcal{G}_{\phi}$ :

$$
*: \operatorname{Fun}\left(\mathcal{G}_{\phi}\right)^{*} \times \operatorname{Fun}\left(\mathcal{G}_{\phi}\right)^{*} \rightarrow \operatorname{Fun}\left(\mathcal{G}_{\phi}\right)^{*}
$$

by the usual formula

$$
(\mu * \nu)(f)=\int_{\mathcal{G}_{\phi} \times \mathcal{G}_{\phi}} f(g h) d \mu(g) d \nu(h)
$$

and one has the bound

$$
\|\mu * \nu\| \leq\|\mu\|\|\nu\|
$$

If $\phi \hookrightarrow \psi$ there is a natural group homomorphism $\mathcal{G}_{\psi} \rightarrow \mathcal{G}_{\phi}$, hence a homomorphism of convolution algebras $\operatorname{Fun}\left(\mathcal{G}_{\psi}\right)^{*} \rightarrow \operatorname{Fun}\left(\mathcal{G}_{\phi}\right)^{*}$. This allows us to give $\operatorname{Fun}_{0}(\mathcal{G})^{*}$, which is the inverse limit of the spaces $\operatorname{Fun}\left(\mathcal{G}_{\psi}\right)^{*}$, the structure of an algebra in a unique way such that all the maps

$$
\operatorname{Fun}_{0}(\mathcal{G})^{*} \rightarrow \operatorname{Fun}\left(\mathcal{G}_{\phi}\right)^{*}
$$

are algebra homomorphisms. We write the product in $\operatorname{Fun}_{0}(\mathcal{G})$ as $*$. Since this product satisfies the bound

$$
\|\mu * \nu\| \leq\|\mu\|\|\nu\|
$$


it extends uniquely by continuity to a product on $\operatorname{Fun}(\mathcal{G})^{*}$, again written $*$ and called the convolution of generalized measures on $\mathcal{G}$.

Moreover, we can average (or convolve) generalized measures on $\mathcal{A}$ against generalized measures on $\mathcal{G}$ as follows. For embedded graph $\phi$ the convolution algebra $\operatorname{Fun}\left(\mathcal{G}_{\phi}\right)^{*} \operatorname{acts}$ on $\operatorname{Fun}\left(\mathcal{A}_{\phi}\right)^{*}$,

$$
*: \operatorname{Fun}\left(\mathcal{G}_{\phi}\right)^{*} \times \operatorname{Fun}\left(\mathcal{A}_{\phi}\right)^{*} \rightarrow \operatorname{Fun}\left(\mathcal{A}_{\phi}\right)^{*}
$$

by the usual formula

$$
(\mu * \nu)(f)=\int_{\mathcal{G}_{\phi} \times \mathcal{A}_{\phi}} f(g A) d \mu(g) d \nu(A)
$$

and one has the bound

$$
\|\mu * \nu\| \leq\|\mu\|\|\nu\|
$$

Using these facts, an inverse limit argument like the one above shows that the convolution algebra $\operatorname{Fun}(\mathcal{G})^{*}$ acts on $\operatorname{Fun}(\mathcal{A})^{*}$. Below, we apply this to construct $\mathcal{G}$-invariant generalized measures on $\mathcal{A}$ from generalized measures on $\mathcal{A}$ by convolution against Haar generalized measure on $\mathcal{G}$ :

Theorem 5. Let $\nu$ be a generalized measure on $\mathcal{A}$ and let $\mu_{\mathrm{H}}$ denote Haar generalized measure on $\mathcal{G}$. Then $\mu_{\mathrm{H}} * \nu$ is a $\mathcal{G}$-invariant generalized measure on $\mathcal{A}$.

Proof - Suppose $f \in \operatorname{Fun}_{0}(\mathcal{A})$. Then $f \in \operatorname{Fun}\left(\mathcal{A}_{\phi}\right)$ for some embedded graph $\phi$, so $f$ depends on $A$ only through $A_{\phi}$; we write $f(A)=F\left(A_{\phi}\right)$. Let $\nu_{\phi}$ be the restriction of $\nu$ to $\operatorname{Fun}\left(\mathcal{A}_{\phi}\right)$. Writing $\mu_{\phi}$ for Haar measure on $\mathcal{G}_{\phi}$, we have, for any $g \in \mathcal{G}$,

$$
\begin{aligned}
\left(\mu_{\mathrm{H}} * \nu\right)(g f) & =\left(\mu_{\phi} * \nu_{\phi}\right)\left(g_{\phi} F\right) \\
& =\int_{\mathcal{G}_{\phi} \times \mathcal{A}_{\phi}} F\left(g_{\phi}^{-1} h A_{\phi}\right) d \mu_{\phi}(h) d \nu_{\phi}\left(A_{\phi}\right) \\
& =\int_{\mathcal{G}_{\phi} \times \mathcal{A}_{\phi}} F\left(h A_{\phi}\right) d \mu_{\phi}(h) d \nu_{\phi}\left(A_{\phi}\right) \\
& =\left(\mu_{\phi} * \nu_{\phi}\right)(F) \\
& =\left(\mu_{\mathrm{H}} * \nu\right)(f) .
\end{aligned}
$$

Since $\operatorname{Fun}_{0}(\mathcal{A})$ is dense in $\operatorname{Fun}(\mathcal{A})$, we conclude that $\left(\mu_{\mathrm{H}} * \nu\right)(g f)=\left(\mu_{\mathrm{H}} * \nu\right)(f)$ for all $f \in \operatorname{Fun}(\mathcal{A})$, so $\mu_{\mathrm{H}} * \nu$ is $\mathcal{G}$-invariant.

\section{Conclusions}

There is much more one can do to generalize the theory of Lie groups and homogeneous spaces to groups of gauge transformations and spaces of connections, using the framework introduced here. For example, there is a Hilbert space completion 
$L^{2}\left(\mathcal{A}, \mu_{u}\right)$ of $\operatorname{Fun}(\mathcal{A})$, on which $\operatorname{Aut}(P)$ has a unitary representation. Similarly, there is a Hilbert space $L^{2}\left(\mathcal{A} / \mathcal{G}, \mu_{\mathrm{AL}}\right)$ on which $\operatorname{Diff}(M)$ has a unitary representation, and a Hilbert space $L^{2}\left(\mathcal{G}, \mu_{\mathrm{H}}\right)$ which is a unitary representation of $\mathcal{G}$ acting by left (or right) translation, as well as a unitary representation of $\operatorname{Aut}(P)$. It is still unclear how useful these structures will be in physics, but they have many of the properties one would naively expect of nonrigorous constructions using "Lebesgue measure" on $\mathcal{A}$ and "Haar measure" on $\mathcal{G}$.

\section{References}

[1] A. Ashtekar and C. J. Isham, Representations of the holonomy algebra of gravity and non-abelian gauge theories, Jour. Class. and Quant. Grav. 9 (1992), 10691100 .

[2] A. Ashtekar and J. Lewandowski, Representation theory of analytic holonomy C*-Algebras, to appear in Knots and Quantum Gravity, ed. J. Baez, Oxford U. Press.

[3] J. Baez, Diffeomorphism-invariant generalized measures on the space of connections modulo gauge transformations, to appear in the proceedings of the Conference on Quantum Topology, eds. L. Crane and D. Yetter, hep-th/9305045.

[4] J. Baez, Link invariants, holonomy algebras and functional integration, U. C. Riverside preprint (1993), hep-th/9301063.

[5] J. Baez, I. Segal and Z. Zhou, An Introduction to Algebraic and Constructive Quantum Field Theory, Princeton U. Press, Princeton, 1992.

[6] A. N. Kolmogorov, Foundations of the Theory of Probability, Chelsea, New York, 1956.

[7] R. Loll, Chromodynamics and gravity as theories on loop space, Penn State University preprint (1993) CGPG-93/9-1, hep-th 9309056.

[8] C. Rovelli and L. Smolin, Loop representation for quantum general relativity, Nucl. Phys. B331 (1990), 80-152. 